\title{
bronchitis and pneumonia
}

Sci. Tsuneo Ishida

2-3-6, Saido, Midori-Ku, Saitama-Shi, Saitama-Ken, †336-0907, Japan

*Corresponding Author: Sci. Tsuneo Ishida, 2-3-6, Saido, Midori-Ku, Saitama-Shi, Saitama-Ken, T 336-0907, Japan.

Received date: July 01, 2020; Accepted date: July 25, 2020; Published date: July 31, 2020

Citation: Sci. Tsuneo Ishida. (2020) Bats: Zinc (II )-immune pediatric virucidal activities for 2019-nCoV prevention and therapeutic effects of COVID-19 bronchitis and pneumonia. Biomedical Research and Clinical Reviews. 1(2); DOI: 10.31579/2692-9406/012

Copyright: $\mathbf{0 2 0 2 0}$ Sci. Tsuneo Ishida, This is an open-access article distributed under the terms of the Creative Commons Attribution License, which permits unrestricted use, distribution, and reproduction in any medium, provided the original author and source are credited.

\section{Abstract:}

Zinc ( II )-immune pediatric virucidal activities for children with 2019-nCoV prevention and COVID-19 bronchitis and pneumonia are discussed, and these $\mathrm{Zn}^{2+}$-immune pediatric virucidal effects result in the following.

Zinc intakes by zinc induced immunity are required $3 \mathrm{mg} /$ day for 7 month to 3 years, $5 \mathrm{mg} /$ day for $4 \sim 8$ years, and $8 \mathrm{mg} / \mathrm{day}$ for 9 $\sim 13$ years in children. Zinc supplementation have been assessed, from $15 \mathrm{mg}$ to $140 \mathrm{mg} /$ week, with the upper range exceeding the recommended daily infection (RDI) for children of $2 \mathrm{mg} /$ day for children less than one year of age and up to $7 \mathrm{mg} / \mathrm{day}$ for children between 1 to 3 years.

Zinc induced pediatric preventing respiratory $2019-\mathrm{nCoV}$ is required that supplementation with zinc gluconate $20 \mathrm{mg}$ in $\mathrm{Zn}$ deficient children resulted in a nearly twofold reduction of acute lower respiratory infections as well as the time to recovery. Zinc supplementation in children is associated with a reduction in the incidence and prevalence of pneumonia. Preventing 2019-nCoV pneumonia is required that zinc supplementation alone $(10$ to $20 \mathrm{mg})$ for more than 3 months significantly reduces in the rate of pneumonia.

Prophylactic zinc supplementation for two weeks may reduce the morbidity due to acute lower respiratory infections. Zinc gluconate supplement may result in significant reduction in respiratory morbidity among children with acute lower respiratory infections. In addition. as serum zinc level of Acute Respiratory Infection (ARI)-children was very low, higher zinc concentration treatments as low sociodemographic factors, poor nutritional status and male children were prevalently associated with higher incidence of acute respiratory infections.

Adjuvant treatment with $20 \mathrm{mg}$ zinc/day accelerates recovery from severe pneumonia in children. Primary outcome was recovery from pneumonia and $30 \mathrm{mg} /$ day of zinc supplementation reduces pneumonia in children with chronic kidney disease (CKD). Zinc supplementation + Chloroquine (CQ)/hydroxychloroquine (HCQ) may be more effective in reducing COVID-19 morbidity and mortality than CQ or HCQ in monotherapy. The serum zinc level returned to a normal level (median, $53.20 \mu \mathrm{mol} / \mathrm{L}$ ) on day $12 \pm 2 \mathrm{in}$ the treatment. There was no statistical difference in the pediatric critic illness score, lung injury score, length of hospital stay, and duration of mechanical ventilation between the zinc treatments. The oxidative stress in pediatric diseases causes an oxidative burst that results in a respiratory burst and rapid ROS production.

Thus, zinc pediatric intake may be required to be effective range $10 \sim 20 \mathrm{mg} / \mathrm{d}$ for $2019-\mathrm{CoV}$ prevention, $10 \sim 30 \mathrm{mg} / \mathrm{d}$ for reduction of COVID-19 bronchitis, and 20 $30 \mathrm{mg} / \mathrm{d}$ for recovery from COVID-19 pneumonia, in which $\mathrm{Zn}^{2+}$ could bind with viral surface proteins by $\mathrm{Zn}^{2+}$ ions-centered tetrahedrally coordination pattern.

Keywords: zinc-induced immune pediatric intake; 2019-cov prevention; covid-19 bronchitis and pneumonia; serum zinc level; $\mathrm{zn}^{2+}$-coordinated pattern

\section{Abbreviations:}

ARI=acute respiratory infection, ARDS=acute respiratory distress syndrome, ALRI=acute lower respiratory infection, $\mathbf{B C G}=$ Bacille Calmette-Guerin, $\mathbf{C Q}=$ Chloroquine, $\mathbf{C K D}=$ chronic kidney disease, COVID-19=Coronavitrus disease-19, $\mathbf{H C Q}=$ hydroxychloroquine,
MERS= Middle East Respiratory Syndrome, NADPH= nicotinamide adenine dinucleotide phosphate, Nox=NADPH oxidases, PICU=pediatric intensive care unit, $\mathbf{R D I}=$ recommended daily intake, $\mathbf{R O S}=$ reactive oxygen species, $\mathbf{R R}=$ Risk Ratio, SARS=Severe Acute Respiratory Syndrome, SARS-CoV-2=Severe Acute Respiratory Syndrome-Coronavirus-2, $\mathbf{X O}=$ xanthine oxidase. 


\section{Introduction}

Epidemiological characteristics and clinical features of pediatric patients with coronaviruses disease-19 (COVID-19) infection are exhibited that the infected children had coinfection with other common respiratory pathogens and the pediatric patients have prolonged fecal shedding of Severe Acute Respiratory Syndrome-Coronavirus-2 (SARS-CoV-2) RNA during the convalescent phase that most pediatric patients had relatively mild disease with good prognosis, which could be seen in children infected with SARS-CoV respiratory viruses with innate immunity in response to pathogen [1]. COVID-19 in children is relatively mild that it is easy to miss the diagnosis in the early stages when present with a non-respiratory disease. The other, severe COVID-19 can also occur in children with underlying or coexisting diseases that the possibility of SARS-CoV-2 infection should be suspected when children show digestive tract symptoms, especially with a severe systemic inflammatory reaction, fever and/or exposure history [2]. Pediatric cases of coronavirus disease 2019 (COVID-19) caused by severe acute respiratory syndrome coronavirus 2 are relatively fewer cases of COVID19 among children compared to cases among adult patients. The mild disease in children may be related to trained immunity that refers to the use of certain vaccines such as Bacille Calmette-Guerin (BCG) to train innate immunity to generate immune memory [3]. On the case of severe pediatric COVID-19 infection, severe thrombocytopenia and acute respiratory distress syndrome (ARDS) are presented, including patient's severe disease course was associated with thrombocytopenia and elevated inflammatory markers. The patient's severe respiratory disease did not improve on IVIg, steroids, azithromycin, and hydroxychloroquine that hyperin flammation may be important in the pathophysiology of COVID19 severe acute respiratory syndrome. Finally, randomized placebo controlled clinical trials was used to study drugs like tocilizumab and remdesivir to include children in addition to adults with COVID-19 [4].

On the other hand, zinc utilization to pediatric antivitus activity becomes effective for childrrn health that zinc-binding proteins such as the metallothioneins may possess antiviral roles, although their specific function remains uncertain [5]. Zinc deficiency is associated with increased respiratory morbidity. Zinc is an antioxidant that protects cells from the damaging effects of oxygen radicals generated during immune activation. The adverse effects of zinc deficiency on the immune system are likely to increase the susceptibility of children and lead to a zincdeficient state. Therefore, zinc supplementation could conceivably modulate the immune and inflammatory responses to viruses in a way that are beneficial to the host [6]. The demonstrated preventive therapeutic effect of zinc in treatment of childhood pneumonia is conflicting. Despite the earlier observed reduction of treatment failure risk and case fatality in children with severe pneumonia, a more recent study demonstrated that $\mathrm{Zn}$ supplementation in 2-24 months old children with radiologically verified pneumonia did not result in significant improvement of risk reduction of treatment failure. Moreover, $\mathrm{Zn}$ supplementation in $\mathrm{Zn}$ deficient children with pneumonia until achievement of normal serum $\mathrm{Zn}$ levels did not improve clinical appearance of the disease [7].

In this mini-review, firstly, zinc induced intake-immune children are described, secondly zinc-immune peridiatic virucidal activities for 2019nCoV prevention, COVID-19 respiratory ailment and pneumonia are discussed, and lastly, zinc induced ROS generation in COVID-19 infection is argued, including with molecular mechsnism which $\mathrm{Zn}^{2+}$ ions could bind with COVID-19 RNA viral surface proteins by $\mathrm{Zn}^{2+}$-centered tetrahedrally coordination pattern.

\section{Zinc-induced immune children}

Zinc intakes by zinc induced immunity are known to be required $3 \mathrm{mg} /$ day for 7 month to 3 years, $5 \mathrm{mg} /$ day for $4 \sim 8$ years, and $8 \mathrm{mg} /$ day for $9 \sim$ 13 years in children. Zinc deficiency was very mild ( 3 to $5.0 \mathrm{mg} \mathrm{Zn}$ intake during the zinc-restricted period), the plasma zinc concentration remained more or less within the normal range and it decreased only after $4 \sim 5$ months of zinc restriction. The other, zinc concentrations in lymphocytes, granulocytes, and platelets decreased within $8 \sim 12$ weeks, suggesting that the assay of cellular zinc provided a more sensitive criterion for diagnosing mild deficiency of zinc, in which zinc enhances the upregulation of mRNA, which in both young adults and elderly subjects, zinc supplementation decreased oxidative stress markers and generation of inflammatory cytokines [8]. A range of zinc supplementation has been assessed, from $15 \mathrm{mg}$ to $140 \mathrm{mg} / \mathrm{week}$, with the upper range exceeding the recommended daily intake (RDI) for children of $2 \mathrm{mg} /$ day for children less than one year of age and up to $7 \mathrm{mg} /$ day for children between 1 to 3 years [9].

\section{Zinc-immune pediatric prevention for respiratory ailment and pneumonia against 2019-nCoV infection}

$\mathrm{Zn}$ supplementation significantly decreased the incidence of acute lower respiratory infection (ALRI) defined according to specific clinical criteria in children aged $<5$ years that zinc reduced childhood with ALRI, but the effect was null if lower specificity case definitions were applied. However, the factor was remained unexplained [10]

Zinc induced pediatric preventing respiratory $2019-\mathrm{nCoV}$ is required as supplementation with $10 \mathrm{mg}$ zinc gluconate in $\mathrm{Zn}$ deficient children resulted in a nearly twofold reduction of the number of episodes of acute lower respiratory infections as well as the time to recovery [11]. Among paediatric populations zinc supplementation for more than 3 months could be effective in preventing pneumonia in children younger than 5 years of age, although the evidence was not robust enough to advocate prophylactic properties if given for shorter periods of time [12]. Given the rising burden of child mortality due to respiratory infections, particularly pneumonia, and considering its decreasing impact with zinc supplementation, further reviews should be considered in which the effectiveness of zinc supplementation should be assessed for acute pneumonia provided that cases are well-defined by strict clinical criteria. Thus, zinc supplementation in children is associated with a reduction in the incidence and prevalence of pneumonia [13].

Preventing pneumonia is required that zinc supplementation alone (10 to $20 \mathrm{mg}$ ), for more than 3 months, was associated with a significant reduction in the rate of pneumonia by $19 \%$, with Risk Ratio (RR) of 0.81 (95\% CI 0.73 to 0.90$)$. Mortality was not statistically different $(\mathrm{RR}=$ $0.85 ; 95 \%$ CI 0.65 to 1.11$)$ [14].

\section{Antiviral avtivity of $\mathrm{Zn2+}$ ions for the children with respiratory COVID-19 infection}

The role of zinc for adult patients with 2019-nCoV infection may be chiefly efficient at the preventions of 2019-CoV, COVID-19 bronchitis and pneumonia, and at the recovery from COVID-19 acute inflammatory lung [15]. However, the influence of zinc on children patients with 2019$\mathrm{nCoV}$ infection may be not necessarily simmilar to adult patients.

$\mathrm{Zn}$ supplementation of $30 \mathrm{mg} /$ day in Thai children reduced significantly severity of acute lower respiratory tract infections resulting in faster disease cessation and shorter hospital stay [16]. 
A decrease of $15 \%(0.78-0.94)$ in days and $12 \%(0.78-0.94)$ in duration of episode in acute respiratory infections was observed. Incidence of acute lower respiratory infections decreased by $62 \%(0.26-0.36)$ and the effect remained for full five months of follow up. Prophylactic zinc supplementation for two weeks may reduce the morbidity due to acute lower respiratory infections but not overall rate of acute respiratory infections in infants aged 6 11 months in similar populations [17]. Effectiveness of zinc gluconate supplementation for 2 months period compared to placebo in reducing respiratory morbidity in acute lower respiratory infected children up to 5 years of age living in zinc poor population. Zinc supplement may result in significant reduction in respiratory morbidity among children with acute lower respiratory infections [11].

Serum zinc level was very low $(25.19 \pm 15.49 \mu \mathrm{gmol} / \mathrm{L})$ with acute respiratory infection (ARI) children as compared that $(55.51 \pm 31.15$ $\mu \mathrm{gmol} / \mathrm{L}$ ) with non-ARI children, in which environment and nutritional status were found to be prevalently associated with higher incidence of acute respiratory infections and serum zinc content had been varied with corresponding sociodemograpic, nutritional and health care profile [18].

\section{Antiviral avtivity of $\mathrm{Zn2+}$ ions for the children with COVID- 19 pneumonia}

Pneumonia is one of the most common implications of lower respiratory tract involvement. The pneumonia is an inflammation on pulmonary parenchyma resulting in exutative solidification of pulmonary tissue of the effect of zinc on the clinical course of pneumoniain 3 to 60-month-old children hospitalized in pediatric wards. Adjuvant treatment with $20 \mathrm{mg}$ zinc per day accelerates recovery from severe pneumonia in children, and could help reduce antimicrobial resistance by decreasing multiple antibiotic exposures, and lessen complications and deaths where second line drugs are unavailable. The mean reduction is equivalent to 1 hospital day for both severe pneumonia and time in hospital. All effects were greater when children with wheezing were omitted from the analysis. 20 mg zinc per day can accelerate the recovery from severe pneumonia in children [19].

The effect of zinc on clinical course of 3 to 60-month children hospitalized due to pneumonia was assumed that this element (zinc) was effective in resolving clinical symptoms and duration of hospitalization. Zinc supplements given during an acute episode are not beneficial in short-term clinical recovery from severe pneumonia in hospitalized children. Primary outcome was recovery from pneumonia which included the incidence and resolving clinical symptoms and duration of hospitalization [20]. Zinc may bebene-ficial for nutritional status in children and adolescent with chronic kidney disease (CKD) due to the fact that participants may have improved their nutritional status through the slight but significant gain in their body mass, especially with $30 \mathrm{mg} /$ day of zinc supplementation. $30 \mathrm{mg} /$ day of zinc supplementation reduces pneumonia in children with CKD [21]. Zinc + Chloroquine (CQ)/ hydroxychloroquine (HCQ) may be more effective in reducing COVID19 morbidity and mortality than CQ or HCQ in monotherapy [22]. The serum zinc level returned to a normal level (median, $53.20 \mu \mathrm{mol} / \mathrm{L}$ ) on day $12 \pm 2$ in the treatment. There was no statistical difference in the pediatric critic illness score, lung injury score, length of hospital stay, and duration of mechanical ventilation between the zinc treatments [23]. The mean serum zinc in patients was normal $(80.77+25.3 \mu \mathrm{g} / \mathrm{dL})$ yet, the mean serum zinc level in pediatric intensive care unit (PICU) patients was lower than that of general ward patients that the lower the serum zinc level, the higher the grade of respiratory distress among children with pneumonia [24]. Moreover, treatment of zinc sulfate plus hydroxychloroquine may play a role in therapeutic management for COVID-19 petients without PICU [25].

\section{Zinc-induced ROS generation in COVID-19 respiratory ailment and pneumonia}

Respiratory viruses are known to induce reactive oxygen species (ROS)generating enzymes, including nicotinamide adenine dinucleotide phosphate oxidases (NADPH oxidases, Nox) and xanthine oxidase (XO) and to disturb antioxidant defenses. ROS generation can induce cell death and the release of virions representing possible proviral role of enhanced ROS production and altered redox balance. The oxidative stress is an antiviral immune response. Too strong immune responses lead to a cytokine storm and severe inflammation, which may disturb lung function and antioxidant supplementation is expected to ameliorate the consequences of infection [26].

Zinc induced ROS generation in respiratory and pulmonary COVID-19 infected cells is that the univalent reduction of oxygen generates superoxide $\bullet \mathrm{O}_{2}{ }^{-}$, hydrogen peroxide $\mathrm{H}_{2} \mathrm{O}_{2}$, and hydroxyl radicals $\bullet \mathrm{OH}$, all of which are reactive oxygen species (ROS). The ROS production and its elimination by the anti-oxidant defense system in cells is a highly modulated process for maintaining normal physiological function in the body, in which the nicotinamide adenine dinucleotide phosphate (NADPH) oxidases are a group of plasma membrane-associated enzymes which catalyze the production of superoxide $\cdot \mathrm{O}_{2}{ }^{-}$from oxygen by using NADPH as the electron donor. Thus, zinc acts as a potent agent by inhibition of ROS production and inflammation [27]. The oxidative stress in pediatric diseases causes an oxidative burst that results in a respiratory burst and rapid ROS production, including superoxide and hydrogen peroxide [28]. However, ROS production in zinc (II )-immune pediatric patient with COVID-19 bronchitis and pneumonia cannot be elucidated yet.

In addition, the role of zinc to pediatric vaccine plays an important 2019nCoV RNA viral degradation, whether a transcriptional step may be involved in zinc-caused inhibition of vaccinia virus growth, zinc-ions (at lower concentration) could inhibit the infection by viral mRNAs degradation, and zinc-ions could inhibit 2019-nCoV by recruiting both the 5' and 3' mRNA degradation to specifically promote the degradation [29].

As mentioned above, $\mathrm{Zn}^{2+}$ ions-induced pediatric virucidal activities for 2019-nCoV prevention and COVID-19 bronchitis and pneumonia are summarily represented in Table 1 .

In conclusion, zinc pediatric intake may be required to be effective range $10 \sim 20 \mathrm{mg} / \mathrm{d}$ for $2019-\mathrm{CoV}$ prevention, $10 \sim 30 \mathrm{mg} / \mathrm{d}$ for reduction of COVID-19 bronchitis, and 20 $30 \mathrm{mg} / \mathrm{d}$ for recovery from COVID-19 pneumonia, in which the molecular mechanism may possess that $\mathrm{Zn}^{2+}$ ions could bind with viral surface proteins by $\mathrm{Zn}^{2+}$-centered tetrahedrally coordination pattern [30]. 


\begin{tabular}{|c|c|c|c|}
\hline $\begin{array}{l}\mathrm{Zn}^{2+} \\
\text { ions }\end{array}$ & \multicolumn{3}{|c|}{$\begin{array}{l}\mathrm{Zn}^{2+} \text { ions induced pediatric antiviral activites for children } \\
\text { prevention, and repiratory ailment and pulmonary disease } \\
\text { against COVID-19 infection }\end{array}$} \\
\hline & Prevention & Respiratory infection & Inflammatory pneumonia \\
\hline $\mathrm{Zn}^{2+}$ & 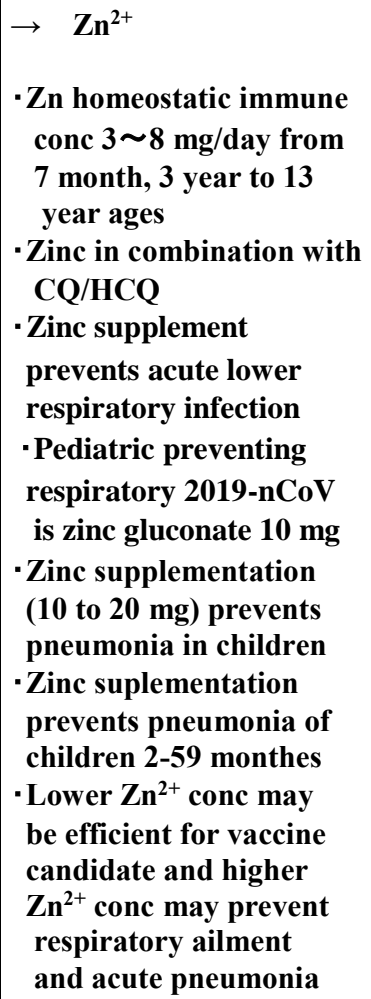 & $\begin{array}{l}\rightarrow \mathrm{Zn}^{2+},\left(\cdot \mathrm{O}_{2}^{-}, \mathrm{H}_{2} \mathrm{O}_{2}, \bullet \mathrm{OH}\right) \\
\cdot \text { Zinc gluconate } 10 \mathrm{mg} \text { in acute } \\
\text { lower respiratory infection } \\
\cdot \mathrm{Zn} \text { supplementation } \\
(30 \mathrm{mg} / \text { day }) \text { in Thai children } \\
\cdot \text { Prophylactic zinc supplemen- } \\
\text { tation in infants aged } 6 \sim 11 \\
\text { months } \\
\cdot \text { Zinc gluconate supplementa- } \\
\text { tion among children with } \\
\text { acute lower respiratory } \\
\text { infections } \\
\cdot \text { Zinc } 15 \text { mg } 30 \text { mg daily with } \\
\text { lozenges providing direct } \\
\text { protective effects in the } \\
\text { upper respiratory tract. }\end{array}$ & $\begin{array}{l}\rightarrow \mathrm{Zn}^{2+},\left(\cdot \mathrm{O}_{2}^{-}, \mathrm{H}_{2} \mathrm{O}_{2}, \bullet \mathrm{OH}\right) \\
\cdot \text { Adjuvant treatment with } \\
20 \mathrm{mg} \text { zinc per day } \\
\cdot \text { Normal }(80.77+25.3 \mu \mathrm{g} / \mathrm{dL}) \\
\cdot \text { Adjuvant } \mathrm{Zinc} \text { Therapy on } \\
\text { Recovery from Pneumonia } \\
\cdot \text { Lower the serum zinc level, } \\
\text { higher the grade of respiratory } \\
\text { distress with children pneumonia } \\
\cdot 30 \text { mg/day of zinc supplemen- } \\
\text { tation reduces pneumonia in } \\
\text { children with CKD } \\
\cdot \text { Zn + CQ/HCQ inhibit } \\
\text { COVID-19 infection } \\
\text { - Zinc supplements during an } \\
\text { acute episode are not beneficial } \\
\text { in short-term clinical recovery } \\
\text { from severe pneumonia in } \\
\text { hospitalized children. }\end{array}$ \\
\hline
\end{tabular}

Table 1: $\mathrm{Zn}^{2+}$ ions-induced pediatric virucidal activities for children prevention, respiratory ailment and pulmonary disease against COVID-

\section{Conclusions}

Zinc ( II )-immune pediatric virucidal activities for children with 2019nCoV prevention and therapeutic effect of COVID-19 bronchitis and pneumonia are discussed, and these pediatric virucidal activity effects result in the following.

Zinc intakes by zinc induced immunity are required $3 \mathrm{mg} /$ day for 7 month to 3 years, $5 \mathrm{mg}$ /day for $4 \sim 8$ years, and $8 \mathrm{mg}$ /day for $9 \sim 13$ years in children. Supplementation have also been assessed, from $15 \mathrm{mg}$ to 140 $\mathrm{mg} /$ week, with the upper range exceeding the RDI for children of 2 $\mathrm{mg}$ /day for children less than one year of age and up to $7 \mathrm{mg} /$ day for children between 1 to 3 years.

Zinc deficiency was very mild (3 to $5.0 \mathrm{mg} \mathrm{Zn}$ intake during the zincrestricted period), the plasma zinc concentration remained more or less within the normal range and it decreased only after $4 \sim 5$ months of zinc restriction. On the other hand, zinc concent-rations in lymphocytes, granulocytes, and platelets decreased within $8 \sim 12$ weeks, suggesting that the assay of cellular zinc provided a more sensitive criterion for diagnosing mild deficiency of zinc, in which zinc enhances the upregulation of mRNA, which in both young adults and elderly subjects,zinc supplementation decreased oxidative stress markers and generation of inflammatory cytokines.
Zinc supplementation reduced the incidence of childhood with ALRI defined by relatively specific clinical criteria, but the effect was null if lower specificity case definitions were applied. The choice of ALRI case definition may substantially influence inferences from community trials regarding the efficacy of preventive interventions. Zinc induced pediatric preventing respiratory $2019-\mathrm{nCoV}$ is that specifically, supplementation with zinc gluconate $10 \mathrm{mg}$ in $\mathrm{Zn}$ deficient children resulted in a nearly twofold reduction of the number of episodes of acute lower respiratory infections as well as the time to recovery. Zinc supplementation in children is associated with a reduction in the incidence and prevalence of pneumonia.

Preventing pneumonia is required that zinc supplementation alone (10 to $20 \mathrm{mg}$ ), for more than 3 months, was associated with a significant reduction in the rate of pneumonia by $19 \%$, with an RR of 0.81 (95\% CI 0.73 to 0.90 ) when compared with the control group (placebo, vitamins, other minerals).

Supplementation (30 mg/day) in Thai children significantly reduced severity of acute lower respiratory tract infections resulting in faster disease cessation and shorter hospital stay. Prophylactic zinc supplementation for two weeks may reduce the morbidity due to acute lower respiratory infections but not overall rate of acute respiratory infections in infants aged 6 11 months in similar populations. Zinc 
gluconate may result in significant reduction in respiratory morbidity among children with acute lower respiratory infections in zinc poor population. In addition. as serum zinc level of Acute Respiratory Infection (ARI)-children was very low, higher zinc concentration treatments as low sociodemographic factors, poor nutritional status and male children were prevalently associated with higher incidence of acute respiratory infections.

Pneumonia is an inflammation on pulmonary parenchyma resulting in exutative solidification of pulmonary tissue, having the effect of zinc on the clinical course of pneumoniain 3 to 60-month-old children hospitalized in pediatric wards. Adjuvant treatment with $20 \mathrm{mg}$ zinc per day accelerates recovery from severe pneumonia in children. Primary outcome was recovery from pneumonia which included the incidence and resolving clinical symptoms and duration of hospitalization. Zinc supplementation 30mg/day reduces pneumonia in children with CKD. Zinc + CQ/HCQ may be more effective in reducing COVID-19 morbidity and mortality than CQ or HCQ in monotherapy.

The serum zinc level returned to a normal level (median, $53.20 \mu \mathrm{mol} / \mathrm{L}$ ) on day $12 \pm 2$ in the treatment. There was no statistical difference in the pediatric critic illness score, lung injury score, length of hospital stay, and duration of mechanical ventilation between the zinc treatments. Respiratory viruses are known to induce ROS-generating enzymes and lead to an oxidative stress that the oxidative stress in pediatric diseases causes an oxidative burst that results in a respiratory burst and rapid ROS production, including superoxide and hydrogen peroxide.

Accordingly, zinc pediatric intake may be required to be effective range $10 \sim 20 \mathrm{mg} / \mathrm{d}$ for 2019-CoV prevention, $10 \sim 30 \mathrm{mg} / \mathrm{d}$ for reduction of COVID-19 bronchitis, and 20 30 mg/d for recovery from COVID-19 pneumonia, in which the molecular mechanism may possess that $\mathrm{Zn}^{2+}$ ions could bind with viral surface proteins by $\mathrm{Zn}^{2+}$-centered tetrahedrally coordination pattern.

\section{Conflicts of Interest}

The author declares there is no conflicts of interest.

\section{Sources of funding}

\section{None}

\section{References}

1. https://pediatrics.aappublications.org/content/pediatrics/early/2 020/05/04/peds.2020-

0961.full.pdf?\&utm_campaign=social\&utm_source=custom_a lert\&utm_medium $=$ pediatrics\&utm_content $=$ clinical_feautres covid\&utm_term=5_9_20

2. X Xiaofang Cai, Yaoling Ma1, Songbo Li, Yan Chen, Zhihui Rong and Wenbin Li. (2020) Clinical characteristics of 5 COVID-19 cases with Non-respiratory Symptoms as the First Manifestation in Children. Frontiers Pediatrics.8, Article 258: 1-9.

3. Q. Cao, Y-C. Chen, C-L. Chen, et al. (2020) SARS-CoV-2 infection in children: Transmission dynamics and clinical characteristics. Journal of the Formosan Medical Association.119: 670-673.

4. Pratik A. Patel, Shanmuganathan Chandrakasan, Geoffrey E. Mickells, Inci Yildirim, et al. (2020) Severe Pediatric COVID19 Presenting With Respiratory Failure and Severe Thrombocytopenia. PEDIATRICS. 146, No. 1; 1-8. DOI:https:// doi.org/10.1542/peds.2020-1437
5. S. A. Read, S. Obeid, C. Ahlenstiel, and G. Ahlenstiel. (2019) The Role of Zinc in Antiviral Immunity. Advances in Nutrition. 10: 696-710.

6. M Dardenne. (2002) Zinc and immune function. European Journal of Clinical Nutrition 56, Suppl 3: S20-S23.

7. A. V. SKALN, L. RINK, O. AJSUVAKOVA, M. ASCHNER, V. A. RITSENKO, et al. (2020) Zinc and respiratory tract infections: Perspectives for COVID-19 (Review). International Journal of Molecular Medicine. 46: 17-26.

8. Ananda S Prasad. (2008) Zinc in Human Health: Effect of Zinc on Immune Cells. MOL MED. 14(5-6) May-June: 353-357.

9. WHO technical staff. (2011) Zinc supplementation to improve treatment outcomes among children diagnosed with respiratory infections. E-Library of Evidence for Nutrition Actions (eLENA).

10. D. E. Roth, S. A. Richard, R. E. Black. (2010) Zinc Supplementation for the Prevention of Acute Lower Respiratory Infection in Children in Developing Countries: Meta-Analysis and Meta-Regression of Randomized Trials. Int J Epidemiol. Jun, 39(3):795-808. Doi: 10.1093/ije/dyp391.

11. U. H. Shah, A.K. Abu-Shaheen, M. A. Malik, S. Alam, M. Riaz, M. A. Al-Tannir. (2013) The efficacy of zinc supplementation in young children with acute lower respiratory infections: A randomized double-blind controlled trial. Clin Nutr. 32(2): 193199. doi: 10.1016/j.clnu.2012.08.018. Epub 2012 Aug 31.

12. Emma Derbyshire, Joanne Delange. (2020) COVID-19: is there a role for immuno-nutrition, particularly in the over $65 \mathrm{~s}$ ? BMJ. Nutrition Prevention \& Health. 2020; 0:1-6. Doi: 10.1136/bmjnph-2020-000071.

13. Zohra S Lassi, Anoosh Moin, Zulfiqar A Bhutta. (2017) Zinc supplementation for the prevention of pneumonia in children aged 2 months to 59 months. The Cochrane Collaboration. Published by John Wiley \& Sons, Ltd. 2016(12):1-41 pages.

14. Teeranai Sakulchit, Ran D. Goldman. (2017) Zinc supplementation for pediatric pneumonia. Child Health Update. 2017; 63: 763-765. october • octobre 2017 | Canadian Family Physician.63:763-765.

15. Tsuneo Ishida. (2020) Zinc ( II ) immune virucidal activities for 2019-nCoV prevention and COVID-19 respiratory ailment and pneumonia. International Journal of Research Studies in Medical and Health Sciences.5, Issue 6: 21-33.

16. S. Rerksuppaphol and L. Rerksuppaphol. (2019) A Randomized Controlled Trial of Zinc Supplementation in the Treatment of Acute Respiratory Tract Infection in Thai Children. Pediatr Rep.11 (2): 15-20. Doi: 10.4081/ pr.2019.7954.Page 15-20.

17. A. Malik, D. K. Taneja, N. Devasenapathy, K. Rajeshwari. (2014) Zinc Supplementation for Prevention of Acute Respiratory Infections in Infants: A Randomized Controlled Trial. Indian Pediatr Actions. 51(10):780-784. Doi: 10.1007/s13312-014-0503-z.

18. S.N. Islam, Md. M. Kamal, R. Rahmatullah, S. K. S. Sadi, M. Ahsan. (2018) Serum zinc levels in children with acute respiratory infections; Association with sociodemography and nutritional status. Clinical Nutrition Experimental. 22: 11-18.

19. M. Yunus, M. S. Yeasmin, R. E. Black, et al. (2004) Zinc for severe pneumonia in very young children: double-blind placebo-controlled trial. THE LANCET. 363, Issue 9422:16831688. DOI: 10.1016/S0140-6736(04)16252-1 W Abdullah Brooks

20. Mohammad Javad Qasemzadeh, Mahdi Fathi, Maryam Tashvighi. (2014) The effect of Adjuvant Zinc Therapy on Recovery from Pneumonia in Hospitalized Children: A DoubleBlind Randomized Controlled Trial. Scientifica.Article ID 694193, 1-4 pages. 
21. Marlene Fabiola Escobedo-Monge, Guido Ayala-Macedo, Graciela Sakihara, Silvia Peralta, et al. (2019) Effects of Zinc Supplementation on Nutritional Status in Children with Chronic Kidney Disease: A Randomized Trial, Nutrients.11, 2671;1-27. doi:10.3390/nu11112671

22. R. Derwand and M. Scholzb. (2020) Does zinc supplementation enhance the clinical efficacy of chloroquine/hydroxychloroquine to win todays battle against COVID-19? Medical Hypotheses. 142: 1-3.

23. X. Yuaul, Su-Yun Qian, Z. Li \& Zhe-Zhe Zhang. (2016) Effect of zinc supplementation on infants with severe pneumonia. World Journal of Pediatrics.12:pages166-169.

24. H. I. Rady, W. A. Rabie, H. A. Rasslan, A. A. ElAyadi. (2013) Blood zinc levels in children hospitalized with pneumonia: A cross sectional study. Egyptian Journal of Chest Diseases and Tuberculosis.62, Issue 4: 697-700.

25. P. M. Carlucci, T. Ahuja, C. Petrilli, H. Rajagopalan, S. Jones, et al. (2020) Hydroxychloroquine and azithromycin plus zinc vs hydroxychloroquine and azithromycin alone: outcomes in hospitalized COVID-19 patients. MedRxiv. 2020:1-22. doi: https;//doi.org/10.1101/2020.05.02. 20080036

26. O. A. Khomich, S. N. Kochetkov, B. Bartosch, and A. V. Ivanov. (2018) Redox Biology of Respiratory Viral Infections. Viruses. Aug; 10(8): 392-419.

27. Ananda S. Prasad and Bin Bao. (2019) Molecular Mechanisms of Zinc as a Pro-Antioxidant Mediator: Clinical Therapeutic Implications. Antioxidants.8 (6); 1-22. doi: 10.3390/antiox 8060164

28. Ahmet Ayaz. (2015) Oxidative Stress and Overview of Pediatric Disease Biomarkers. Journal of Pediatric Biochemistry. 5, No. 1: 8-11.

29. Tsuneo Ishida. (2019) Anti-Viral Vaccine Activity of Zinc (II ) for Viral Prevention, Entry, Replication, and Spreading During Pathogenesis Process. Current Trends in Biomedical Engineering \& Biosciences. 19 (3):83-87.

30. Tsuneo Ishida. (2019) Review on the Role of $\mathrm{Zn}^{2+}$ Ions in Viral Pathogenesis and the Effect of $\mathrm{Zn}^{2+}$ Ions for Host Cell-Virus Growth Inhibition. American Journal of Biomedical Science \& Research. 2(1): 28-37.

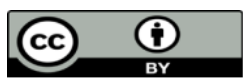

This work is licensed under Creative Commons Attribution 4.0 License

\section{To Submit Your Article Click Here: Submit Article}

DOI: $10.31579 / 2692-9406 / 012$
Ready to submit your research? Choose Auctores and benefit from:

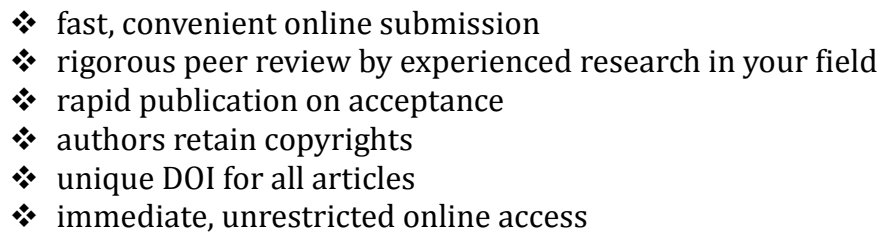

At Auctores, research is always in progress.

Learn more www.auctoresonline.org/journals/biomedical-researchand-clinical-reviews-- 